\title{
Sustained Intraocular Pressure Elevation After Intravitreal Injection of Bevacizumab and Ranibizumab Associated with Trabeculitis
}

\author{
Matthew Sniegowski, Naresh Mandava and Malik Y. Kahook* \\ Rocky Mountain Lions Eye Institute, Department of Ophthalmology, University of Colorado Denver, Aurora, CO 80045 , \\ USA
}

\begin{abstract}
Anti-vascular endothelial growth factor agents are frequently used to treat a variety of ocular neovascular diseases. While agents like bevacizumab and ranibizumab appear to be safe and effective, there have been reports of severe intraocular inflammation as well as sustained elevation of intraocular pressure (IOP) after single or multiple intravitreal injections of these protein-based therapeutics. The true mechanism leading to inflammation and/or sustained spikes in IOP remains unknown. We report a patient with sustained IOP elevation and kerato-precipitates on the trabecular meshwork after multiple injections of both bevacizumab and ranibizumab. We propose that monomer antibodies, aggregated proteins, or other high molecular weight molecules might lead to inflammation in the trabecular meshwork and subsequent elevation in IOP.
\end{abstract}

Keywords: Bevacizumab, ranibizumab, vascular endothelial growth factor, glaucoma, intraocular pressure, neovascular agerelated macular degeneration, trabeculitis, inflammation.

\section{INTRODUCTION}

Intravitreal injections of anti-vascular endothelial growth factor (VEGF) agents are commonly used to treat a variety of retinal and choroidal neovascular diseases. Intra-ocular pressure (IOP) is known to be elevated in the period immediately following an intravitreal injection; however, it was thought that these elevations were transient with a return of IOP to near baseline levels after thirty minutes [1-3]. Recently, an association between intravitreal anti-VEGF injections and sustained IOP elevations has been made [4-6]. Previous reports suggest that a likely mechanism for the prolonged increase in IOP may result from trabecular meshwork blockage due to the large size of bevacizumab (molecular weight $=149 \mathrm{kD}$ ) [4-6]. We present a case of elevated IOP in the setting of a unilateral trabeculitis after anti-VEGF therapy. We propose that an alternative theory for this observed phenomenon is that protein aggregates or other contaminants within the injected fluid may induce an immunologic response with subclinical inflammation and secondary IOP elevation [7].

\section{CASE REPORT}

A 66 year old male with exudative age-related macular degeneration (AMD) in his right eye and non-exudative AMD in his left eye was referred to our tertiary care center for treatment. The patient's ocular history prior to being seen by our retina service was significant for bilateral pseudophakia followed by subsequent laser capsulotomies for bilateral posterior capsular opacification. Over the course of his care, he received three injections of bevacizumab (Avastin, Genentech, Inc., San Francisco, CA) and eighteen

*Address correspondence to this author at the University of Colorado Denver School of Medicine, Department of Ophthalmology, 1675 Aurora Ct, Mail Stop F-731, Aurora, Colorado 80045, USA; Tel: 720-848-2500; Fax: 720848-5014; E-mail: malik.kahook@gmail.com injections of ranibizumab (Lucentis, Genentech, Inc., San Francisco, CA). Previous IOP levels in both eyes were stable in the mid-teens for two years prior to presenting to our clinic. The patient was followed over the next four years and received injections of either ranibizumab or bevacizumab in the right eye at intervals between 4 and 12 weeks. He was noted to have occasional elevations in IOP up to $20-21 \mathrm{~mm}$ $\mathrm{Hg}$, in his right eye, over the first three years of treatment. The left eye IOP remained stable in the low to mid teens. After 12 intravitreal injections, the patient began to have fluctuations in right eye IOP ranging from 22-26 mm $\mathrm{Hg}$. Upon examination twelve weeks after the eighteenth injection of ranibizumab, the patient was found to have an IOP of $32 \mathrm{~mm} \mathrm{Hg}$ on the right side. He was started on brimonidine three times a day and referred to the glaucoma service the next day. Upon follow-up examination, the patient's IOP was found to have decreased to $22 \mathrm{~mm} \mathrm{Hg}$ and gonioscopic examination demonstrated what appeared to be kerato-precipitates on the trabecular meshwork (Figs. 1A, B). Brimonidine was stopped and the patient was started on prednisolone acetate $1 \%$ four times a day. The IOP decreased to $18 \mathrm{~mm} \mathrm{Hg}$ three days later. While the anterior chamber was initially devoid of any inflammatory cells, slight cell and flare appeared on subsequent follow up examinations and then faded with the continued topical steroid therapy. Subsequent work-up for uveitis was negative. The IOP remained low over subsequent visits without use of topical pressure lowering drops and the kerato-precipitates resolved. The patient has been weaned off of the steroid drops without recurrence of inflammation or IOP spikes and we have avoided further use of anti-VEGF agents.

\section{DISCUSSION}

With widespread use of intravitreal anti-VEGF therapy for a variety of ophthalmic diseases, cases of sustained elevation of IOP are beginning to emerge [4-6]. Both bevacizumab, a 149 
$\mathrm{kDa}$ full-length antibody and, ranibizumab, a $48 \mathrm{kDa}$ antibody fragment, are known to diffuse into the anterior chamber, where they persist for some time prior to exiting through the aqueous outflow system [8, 9]. Previously published reports, have suggested that the increased IOP following intravitreal bevacizumab may be, at least in part, secondary to a physical blockage of the outflow system by the anti-VEGF agents. Blockade of the trabecular meshwork outflow system from bevacizumab monomers and fab fragments of ranibizumab is unlikely to be the true reason behind this phenomenon due to the low incidence of reported spikes in IOP. It may be that protein-based therapeutics can occasionally be altered in some way to cause protein aggregation with subsequent blockade of aqueous egress. Another possibility is that contaminants, such as silicone oil from the syringe barrel or rubber stopper, could lead to obstruction of the outflow system [10]. These theories remain speculative at this point.

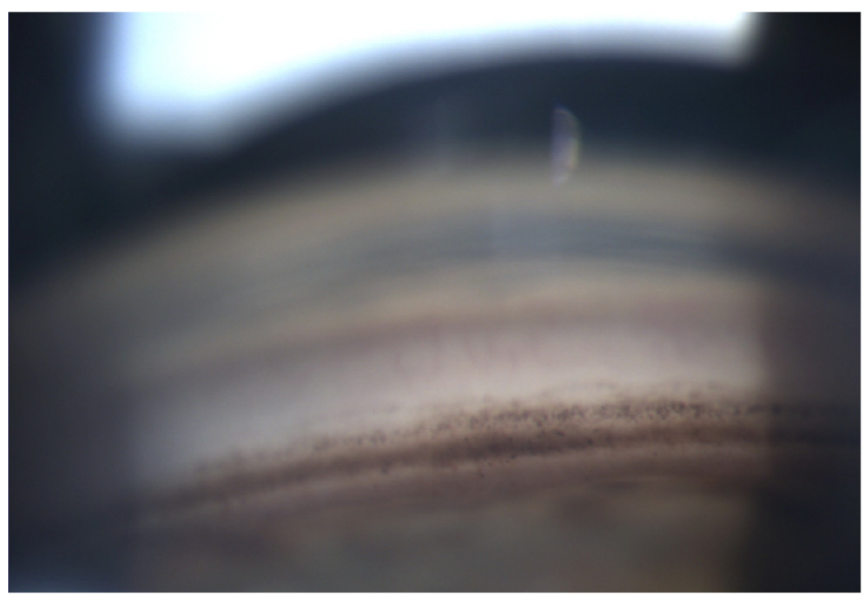

Fig. (1A). Gonioscopic view of the uninvolved left eye showing normal drainage angle anatomy.

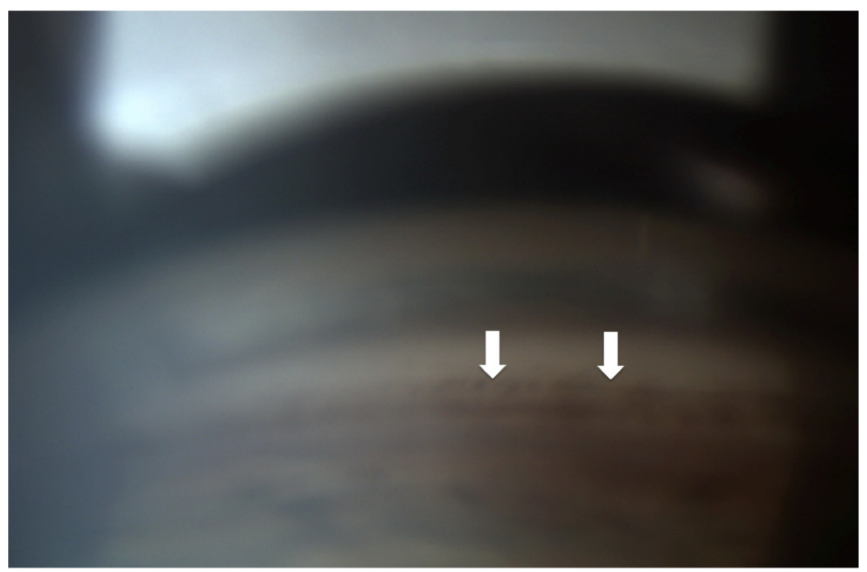

Fig. (1B). Gonioscopic view of the right eye revealing a hazy view with white deposits (arrow) over the trabecular meshwork.

Significant inflammatory reactions, even after previous uneventful injections are known to occur following the intravitreal injections of bevacizumab [11]. It is also well known and characterized that aggregated proteins, especially multimers in excess of $100 \mathrm{kD}$, induce a much more vigorous immunologic response than their non-aggregated counterparts. Even small amounts of foreign protein aggregates induce a much stronger immune response than larger amounts of endogenous protein [7]. The intraocular injection of foreign proteins may lead to an inflammatory reaction and subsequent trabeculitis and IOP elevation in some patients. This phenomenon, along with other possible coexisting factors such as mechanical blockage of the outflow system, suggests that patients should be followed closely for spikes in IOP that can lead to optic nerve damage. While the present case may illustrate a potential link between anti-VEGF injections and subsequent inflammation with IOP elevation, there remains no proof of definitive direct causal relationship for the observed phenomenon. In the case we report, the patient maintained a decrease in IOP after discontinuation of topical aqueous suppressants and initiation of topical steroid therapy. This may have implications for future antibody based therapeutics for ophthalmic diseases. More research is needed in this area to fully elucidate the mechanism of increased intraocular pressure following intravitreal bevacizumab and ranibizumab.

\section{ACKNOWLEDGEMENTS}

Malik Y. Kahook and Naresh Mandava have received research support from Genentech in the past.

\section{REFERENCES}

[1] Landa G, Amde W, Doshi V, et al. Comparative study of intravitreal bevacizumab (Avastin) vs ranibizumab (Lucentis) in the treatment of neovascular age-related macular degeneration. Ophthalmologica 2009; 223(6): 370-5

[2] Kim J, Mantravadi A, Hur E, Covert D. Short-term intraocular pressure changes immediately after intravitreal injections of antivascular endothelial growth factor agents. Am J Ophthalmol 2008; 146: 930-4.

[3] Frenkel R, Mani L, Toler A, Frenkel M. Intraocular pressure effects of pegaptanib (Macugen) injections in patients with and without glaucoma. Am J Ophthalmol 2007; 143: 1034-5.

[4] Jalil A, Fenerty C, Charles S. Intravitreal bevacizumab (Avastin) causing acute glaucoma: an unreported complication. Eye 2007; 21: 1541 .

[5] Kahook M, Kimura A, Wong L, et al. Sustained elevation in intraocular pressure associated with intravitreal bevacizumab injections. Ophthalmic Surg Lasers Imaging 2009; 40: 293-5.

[6] Carver J, Bouska C, Corey R. Avastin and risk of glaucoma. Retina Congress, September $30^{\text {th }}$-October-4th, 2009, NY, (Abstract).

[7] Rosenberg AS. Effects of protein aggregates: an immunologic perspective. AAPS J 2006; 8(3): E501-7.

[8] Bakri S, Synder M, Reid J, et al. Pharmacokinetics of intravitreal ranibizumab (Lucentis). Ophthalmology 2007; 114: 2179-82.

[9] Krohne TU, Eter N, Holz F, Meyer C. Intraocular pharmacokinetics of bevacizumab after a single intravitreal injection in humans. Am J Ophthalmol 2008; 146: 508-12.

[10] Bakri SJ, Ekdawi NS. Intravitreal silicone oil droplets after intravitreal drug injections. Retina 2008; 28(7): 996-1001.

[11] Georgopoulos M, Polak K, Prager F, et al. Characteristics of severe intraocular inflammation following intravitreal injection of bevacizumab (Avastin). Br J Ophthalmol 2009; 93: 457-62. 